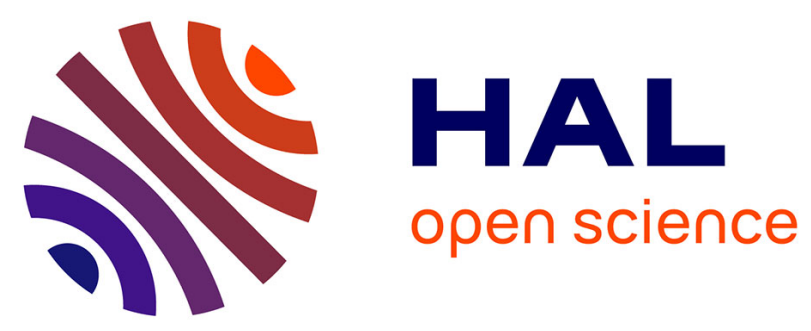

\title{
New trends in deterministic lower bounds and SNR threshold estimation: from derivable bounds to conjectural bounds
}

\author{
Eric Chaumette, Alexandre Renaux, Pascal Larzabal
}

\section{To cite this version:}

Eric Chaumette, Alexandre Renaux, Pascal Larzabal. New trends in deterministic lower bounds and SNR threshold estimation: from derivable bounds to conjectural bounds. Sensor Array Multichannel Workshop SAM 2010, Oct 2010, Kibutz Ma'ale Hahamisha, Israel. hal-02508675

\section{HAL Id: hal-02508675 \\ https://hal.science/hal-02508675}

Submitted on 15 Mar 2020

HAL is a multi-disciplinary open access archive for the deposit and dissemination of scientific research documents, whether they are published or not. The documents may come from teaching and research institutions in France or abroad, or from public or private research centers.
L'archive ouverte pluridisciplinaire HAL, est destinée au dépôt et à la diffusion de documents scientifiques de niveau recherche, publiés ou non, émanant des établissements d'enseignement et de recherche français ou étrangers, des laboratoires publics ou privés. 


\title{
NEW TRENDS IN DETERMINISTIC LOWER BOUNDS AND SNR THRESHOLD ESTIMATION: FROM DERIVABLE BOUNDS TO CONJECTURAL BOUNDS
}

\author{
Eric Chaumette ${ }^{(1)}$, Alexandre Renaux ${ }^{(2)}$ and Pascal Larzabal ${ }^{(3)}$ \\ (1) ONERA - DEMR/TSI, The French Aerospace Lab, Chemin de la Hunière, F-91120 Palaiseau, France \\ (2) Université Paris-Sud 11, L2S, Supelec, 3 rue Joliot Curie, F-91190 Gif-Sur-Yvette, France \\ (3) SATIE, ENS Cachan, CNRS, UniverSud, 61 av President Wilson, F-94230 Cachan, France
}

\begin{abstract}
It is well known that in non-linear estimation problems the ML estimator exhibits a threshold effect, i.e. a rapid deterioration of estimation accuracy below a certain SNR or number of snapshots. This effect is caused by outliers and is not captured by standard tools such as the Cramér-Rao bound (CRB). The search of the SNR threshold value can be achieved with the help of approximations of the Barankin bound (BB) proposed by many authors. These approximations may result from linear or non-linear transformation (discrete or integral) of the uniform unbiasedness constraint introduced by Barankin. Additionally, the strong analogy between derivations of deterministic bounds and Bayesian bounds of the Weiss-Weinstein family has led us to propose a conjectural bound which outperforms existing ones for SNR threshold prediction.
\end{abstract}

Index Terms - Parameter estimation, mean-square-error bounds, SNR threshold

\section{INTRODUCTION}

Minimal performance bounds allow for calculation of the best performance that may be achieved, in the Mean Square Error (MSE) sense, when estimating a set of model parameters from noisy observations. Historically the first MSE lower bound for deterministic parameters to be derived was the Cramér-Rao Bound (CRB) [4], which has been the most widely used since. Its popularity is largely due to its simplicity of calculation leading to closed-form expressions useful for system analysis and design. Additionally, the CRB can be achieved asymptotically (high SNR and/or large number of snapshots) by Maximum Likelihood Estimators (MLE), and last but not least, it is the lowest bound on the MSE of unbiased estimators, since it derives from a local formulation of unbiasedness in the vicinity of the true parameters [2]. This initial characterization of locally unbiased estimators has been improved first by Bhattacharyya's works [4] which refined the characterization of local unbiasedness, and significantly generalized by Barankin works [1], who established the general form of the greatest lower bound on MSE (BB) taking into account a uniform unbiasedness definition (eq. (1)). Unfortunately

This work has been partly funded by the European Network of excellence NEWCOM++ under the number 216715

This project is partialy funded by both the Région Ile-de-France and the Digiteo Research Park the $\mathrm{BB}$ is the solution of an integral equation with a generally incomputable analytic solution (eq. (8)).

Therefore, since then, numerous works detailed in [2][3] have been devoted to deriving computable approximations of the $\mathrm{BB}$ and have shown that the CRB and the BB can be regarded as key representatives of two general classes of bounds, respectively the Small-Error bounds and the Large-Error bounds. These works have also shown that in non-linear estimation problems three distinct regions of operation can be observed. In the asymptotic region, i.e. at a high number of independent snapshots and/or at high SNR, the MSE is small and, in many cases, close to the Small-Error bounds. In the $a$ priori performance region where the number of independent snapshots and/or the SNR are very low, the observations provide little information and the MSE is close to that obtained from the prior knowledge about the problem. Between these two extremes, there is a transition region where MSE of MLEs usually deteriorates rapidly with respect to existing MSE lower bounds (Large or Small) and exhibits a threshold behaviour, which corresponds to a "performance breakdown" of the estimators due to the appearance of outliers.

Small-Error bounds are not able to handle the threshold phenomena, whereas it is revealed by Large-Error bounds that can be used to predict the threshold value. On the other hand, Large-Error bounds suffer from their computational cost. Indeed, each BB approximation request the search of an optimum over a set of test points and their tightness depends on the chosen set of test points.

And tightness is the matter, since a more accurate knowledge of the BB allows a better prediction of the SNR threshold value.

Therefore, at least two strategies can be adopted.

The first one is the most consistent with deductive reasoning applied to the unbiasedness paradigm. This strategy, fully mastered from the mathematics and the meaning point of view, provides derivable bounds and relies on the introduction a general class of possible transformations (eq. (11)) of the uniform unbiasedness constraint (eq. (1)), i.e. the mixture of integral linear and non-linear transformations, opening a wide variety of directions in the search of computable tighter $\mathrm{BB}$ approximations.

The second one is partially consistent with deductive reasoning since it may be based - see our example Section 3 - on analogies between families of lower bounds without the support of a non-questionnable derivation and interpretation. And yet, but nevertheless this strategy may yield some conjectural bounds tightest than the existing and well-estabished ones. 


\section{DERIVABLE LOWER BOUNDS}

\subsection{Linear transformations of the unbiasedness constraint}

For the sake of simplicity we will focus on the estimation of a single real function $g(\theta)$ of a single unknown real deterministic parameter $\theta$. In the following, unless otherwise stated, $\mathbf{x}$ denotes the random observation vector of dimension $M, \Omega$ the observations space, and $p(\mathbf{x} ; \theta)$ the probability density function (p.d.f.) of $\mathbf{x}$ depending on $\theta \in \Theta$, where $\Theta$ denotes the parameter space. Let $L^{2}(\Omega)$ be the real Hilbert space of square integrable functions over $\Omega$.

In the search for a lower bound on the MSE of unbiased estimators, two fundamental properties of the problem at hand, introduced by Barankin [1], must be noticed. The first property is that the MSE of a particular estimator $\widehat{g\left(\theta^{0}\right)}(\mathbf{x}) \in L^{2}(\Omega)$ of $g\left(\theta^{0}\right)$, where $\theta^{0}$ is a selected value of the parameter $\theta$, is a norm associated with a particular scalar product $\langle\mid\rangle_{\theta}$ :

$$
\begin{aligned}
M S E_{\theta^{0}}\left[\widehat{g\left(\theta^{0}\right)}\right] & =\left\|\widehat{g\left(\theta^{0}\right)}(\mathbf{x})-g\left(\theta^{0}\right)\right\|_{\theta^{0}}^{2}, \\
\langle g(\mathbf{x}) \mid h(\mathbf{x})\rangle_{\theta^{0}} & =E_{\theta^{0}}\left[g(\mathbf{x})^{*} h(\mathbf{x})\right] .
\end{aligned}
$$

The second property is that an unbiased estimator $\widehat{g\left(\theta^{0}\right)}(\mathbf{x})$ of $g(\theta)$ should be uniformly unbiased, i.e. for all possible values of the unknown parameter $\theta \in \Theta$ it must satisfy:

$$
E_{\theta}\left[\widehat{g\left(\theta^{0}\right)}(\mathbf{x})\right]=g(\theta)=E_{\theta^{0}}\left[\widehat{g\left(\theta^{0}\right)}(\mathbf{x}) \nu(\mathbf{x} ; \theta)\right],
$$

where $\nu(\mathbf{x} ; \theta)=\frac{p(\mathbf{x} ; \theta)}{p\left(\mathbf{x} ; \theta^{0}\right)}$ denotes the Likelihood Ratio (LR). As a consequence, the locally-best (at $\theta^{0}$ ) unbiased estimator is the solution of a norm minimization under linear constraints

$\min \left\{M S E_{\theta^{0}}\left[\widehat{g\left(\theta^{0}\right)}\right]\right\}$ under $E_{\theta^{0}}\left[\widehat{g\left(\theta^{0}\right)}(\mathbf{x}) \nu(\mathbf{x} ; \theta)\right]=g(\theta)$, solution that can be obtained by using the norm minimization lemma

$$
\begin{gathered}
\min \left\{\mathbf{u}^{H} \mathbf{u} \text { under } \mathbf{c}_{k}^{H} \mathbf{u}=v_{k}, 1 \leq k \leq K\right\}=\mathbf{v}^{H} \mathbf{G}^{-1} \mathbf{v} \\
\mathbf{u}_{o p t}=\sum_{k=1}^{K} \alpha_{k} \mathbf{c}_{k}, \quad \boldsymbol{\alpha}=\mathbf{G}^{-1} \mathbf{v}, \quad \mathbf{G}_{n, k}=\mathbf{c}_{n}^{H} \mathbf{c}_{k}
\end{gathered}
$$

Unfortunately, as shown hereinafter, if $\Theta$ contains a continuous subset of $\mathbb{R}$, then the norm minimization under a set of an infinite number of linear constraints (1) leads to an integral equation (8) with no analytical solution in general. Therefore, since the original work of Barankin [1], many studies [2, and references therein][3] have been dedicated to the derivation of "computable" lower bounds approximating the MSE of the locally-best unbiased estimator (BB). All these approximations derive from sets of discrete or integral linear transform of the "Barankin" constraint (1), and accordingly of the LR, and can be obtained using the following simple rationale. Let $\boldsymbol{\theta}^{N}=\left(\theta^{1}, \ldots, \theta^{N}\right)^{T} \in \Theta^{N}$ be a vector of $N$ test points, $\nu\left(\mathbf{x} ; \boldsymbol{\theta}^{N}\right)=\left(\nu\left(\mathbf{x} ; \theta^{1}\right), \ldots, \nu\left(\mathbf{x} ; \theta^{N}\right)\right)^{T}$ be the vector of LR associated to $\boldsymbol{\theta}^{N}, \xi(\theta)=g(\theta)-g\left(\theta^{0}\right)$ and $\boldsymbol{\xi}\left(\boldsymbol{\theta}^{N}\right)=\left(\xi\left(\theta^{1}\right), \ldots, \xi\right.$ Any unbiased estimator $\widehat{g\left(\theta^{0}\right)}(\mathbf{x})$ satisfying (1) must comply with

$$
E_{\theta^{0}}\left[\left(\widehat{g\left(\theta^{0}\right)}(\mathbf{x})-g\left(\theta^{0}\right)\right) \boldsymbol{\nu}\left(\mathbf{x} ; \boldsymbol{\theta}^{N}\right)\right]=\boldsymbol{\xi}\left(\boldsymbol{\theta}^{N}\right),
$$

and with any subsequent linear transformation of (3). Therefore, any given set of $K(K \leq N)$ independent linear transformations of (3):

$$
E_{\theta^{0}}\left[\left(\widehat{g\left(\theta^{0}\right)}(\mathbf{x})-g\left(\theta^{0}\right)\right) \mathbf{h}_{k}^{T} \boldsymbol{\nu}\left(\mathbf{x} ; \boldsymbol{\theta}^{N}\right)\right]=\mathbf{h}_{k}^{T} \boldsymbol{\xi}\left(\boldsymbol{\theta}^{N}\right),
$$

$\mathbf{h}_{k} \in \mathbb{R}^{N}, 1 \leq k \leq K$, provides with a lower bound on the MSE (2):

$$
M S E_{\theta^{0}}\left[\widehat{g\left(\theta^{0}\right)}\right] \geq \boldsymbol{\xi}\left(\boldsymbol{\theta}^{N}\right)^{T} \widetilde{\mathbf{G}}_{\mathbf{H}_{K}} \boldsymbol{\xi}\left(\boldsymbol{\theta}^{N}\right),
$$

where $\widetilde{\mathbf{G}}_{\mathbf{H}_{K}}=\mathbf{H}_{K}\left(\mathbf{H}_{K}^{T} \mathbf{R}_{\nu} \mathbf{H}_{K}\right)^{-1} \mathbf{H}_{K}^{T}, \mathbf{H}_{K}=\left[\mathbf{h}_{1} \ldots \mathbf{h}_{K}\right]$ and $\left(\mathbf{R}_{\nu}\right)_{n, m}=E_{\theta^{0}}\left[\nu\left(\mathbf{x} ; \theta^{n}\right) \nu\left(\mathbf{x} ; \theta^{m}\right)\right]$. The BB is obtained by taking the supremum of (5) over all the existing degrees of freedom $\left(N, \boldsymbol{\theta}^{N}, K, \mathbf{H}_{K}\right)$. Moreover, for a given vector of test points $\boldsymbol{\theta}^{N}$, the lower bound (5) reaches its maximum iff the matrix $\mathbf{H}_{K}$ is invertible $(K=N)$, which represents a bijective transformation of the set of the $N$ initial constraints (3):

$$
M S E_{\theta^{0}}\left[\widehat{g\left(\theta^{0}\right)}\right] \geq \boldsymbol{\xi}\left(\boldsymbol{\theta}^{N}\right)^{T} \widetilde{\mathbf{G}}_{\mathbf{I}_{N}} \boldsymbol{\xi}\left(\boldsymbol{\theta}^{N}\right) \geq \boldsymbol{\xi}\left(\boldsymbol{\theta}^{N}\right)^{T} \widetilde{\mathbf{G}}_{\mathbf{H}_{K}} \boldsymbol{\xi}\left(\boldsymbol{\theta}^{N}\right),
$$

where $\mathbf{I}_{N}$ is the identity matrix with dimension $N$. All known bounds on the MSE deriving from the Barankin Bound is a particular implementation of (5), including the most general formalism introduced lately in [3]. Indeed, the limit of (4) where $N \rightarrow \infty$ and $\boldsymbol{\theta}^{N}$ uniformly samples $\Theta$ leads to the linear integral constraint:

$$
\begin{gathered}
E_{\theta^{0}}\left[\left(\widehat{g\left(\theta^{0}\right)}(\mathbf{x})-g\left(\theta^{0}\right)\right) \eta(\mathbf{x}, \tau)\right]=\Gamma_{h}(\tau), \\
\eta(\mathbf{x}, \tau)=\int_{\Theta} h(\tau, \theta) \nu(\mathbf{x} ; \theta) d \theta, \quad \Gamma_{h}(\tau)=\int_{\Theta} h(\tau, \theta) \xi(\theta) d \theta,
\end{gathered}
$$

where each $\mathbf{h}_{k}=\left(h\left(\tau_{k}, \theta^{1}\right), \ldots, h\left(\tau_{k}, \theta^{N}\right)\right)^{T}$ is the vector of samples of a parametric function $h(\tau, \theta), \tau \in \Lambda \subset \mathbb{R}$, integrable over $\Theta, \forall \tau \in \Lambda$. Then, for any subset of $K$ values of $\tau$, $\left\{\tau_{k}\right\}_{1<k<K}$, the subset of the associated $K$ linear integral constraints $(\overline{6})$ leads to the following lower bound (2):

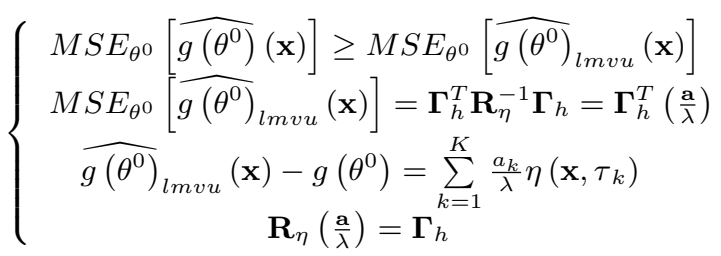

where $\left(\mathbf{R}_{\eta}\right)_{k, k^{\prime}}=E_{\theta^{0}}\left[\eta\left(\mathbf{x}, \tau_{k}\right) \eta\left(\mathbf{x}, \tau_{k^{\prime}}\right)\right]$ and $\left(\boldsymbol{\Gamma}_{h}\right)_{k}=\Gamma\left(\tau_{k}\right)$. Therefore, when $K \rightarrow \infty$ and the set $\left\{\tau_{k}\right\}_{1<k<K}$ uniformly samples $\Lambda$, by setting $\frac{1}{\lambda}=d \tau=\tau_{k+1}-\tau_{k}, \boldsymbol{\beta}=\frac{\mathbf{a}}{\lambda}$, the integral form of the above lower bound appears straightforwardly:

$$
\left\{\begin{array}{l}
M S E_{\theta^{0}}\left[{\widehat{g\left(\theta^{0}\right)}}_{l m v u}(\mathbf{x})\right]=\int_{\Lambda} \Gamma_{h}(\tau) \beta(\tau) d \tau \\
{\widehat{g\left(\theta^{0}\right)}}_{l m v u}(\mathbf{x})-g\left(\theta^{0}\right)=\int_{\Lambda} \eta(\mathbf{x}, \tau) \beta(\tau) d \tau, \\
\int_{\Lambda} K_{h}\left(\tau^{\prime}, \tau\right) \beta(\tau) d \tau=\Gamma_{h}\left(\tau^{\prime}\right)
\end{array},\right.
$$

$$
\begin{aligned}
K_{h}\left(\tau, \tau^{\prime}\right) & =E_{\theta^{0}}\left[\eta(\mathbf{x}, \tau) \eta\left(\mathbf{x}, \tau^{\prime}\right)\right] \\
& =\iint_{\Theta} h(\tau, \theta) R_{\nu}\left(\theta, \theta^{\prime}\right) h\left(\tau^{\prime}, \theta^{\prime}\right) d \theta d \theta^{\prime}, \\
T^{T} R_{\nu}\left(\theta, \theta^{\prime}\right) & =E_{\theta^{0}}\left[\frac{p(\mathbf{x} ; \theta)}{p\left(\mathbf{x} ; \theta^{0}\right)} \frac{p\left(\mathbf{x} ; \theta^{\prime}\right)}{p\left(\mathbf{x} ; \theta^{0}\right)}\right]=\int_{\Omega} \frac{p(\mathbf{x} ; \theta) p\left(\mathbf{x} ; \theta^{\prime}\right)}{p\left(\mathbf{x} ; \theta^{0}\right)} d \mathbf{x},
\end{aligned}
$$

which is exactly the main result introduced in [3] and is a generalization of the Kiefer Bound [4] $(K=2)$. Note that if $h(\tau, \theta)=$ $\delta(\tau-\theta)$ (limit case of $\mathbf{H}_{N}=\mathbf{I}_{N}$ where $N=K \rightarrow \infty$ ) then $K_{h}\left(\tau, \tau^{\prime}\right)=R_{\nu}\left(\tau, \tau^{\prime}\right)$ and (8) becomes the simplest expression of the exact Barankin Bound [2, (10)]. As mentioned above, in most practical cases, it is impossible to find either the limit of (7) or an analytical solution of (8) to obtain an explicit form of the exact Barankin Bound on the MSE, which somewhat limits its interest. 
Nevertheless this formalism allows to use discrete (4) or integral (6) linear transforms of the LR, possibly non-invertible, possibly optimized for a set of p.d.f. (such as the Fourier transform in [3]) in order to get a tight approximation of the BB.

\subsection{Non-linear transformations of the unbiasedness constraint}

Let us consider the set of estimation problems characterized by a p.d.f. for which there exists a real valued function $t$ such that:

$$
t(p(\mathbf{x} ; \theta))=k(\theta, t) p(\mathbf{x} ; \gamma(\theta, t)), \quad k(\theta, t)=\int_{\Omega} t(p(\mathbf{x} ; \theta)) d \mathbf{x}
$$

Then an unbiased estimator satisfying (1) satisfies as well [5], $\forall \theta \in$ $\Theta$ :

$$
\begin{aligned}
E_{\theta^{0}}\left[\left(\widehat{g\left(\theta^{0}\right)}(\mathbf{x})-g\left(\theta^{0}\right)\right)\right. & \left.\frac{t(p(\mathbf{x} ; \theta))}{p\left(\mathbf{x} ; \theta^{0}\right)}\right]= \\
& k(\theta, t)\left[g(\gamma(\theta, t))-g\left(\theta^{0}\right)\right] .
\end{aligned}
$$

Moreover, if there exists a set of functions $t_{\theta}$ satisfying (9), then we can update the definition of $\nu(\mathbf{x} ; \theta)$ and $\xi(\theta)$ in (6) according to:

$\nu(\mathbf{x} ; \theta)=\frac{t_{\theta}(p(\mathbf{x} ; \theta))}{p\left(\mathbf{x} ; \theta^{0}\right)}, \quad \xi(\theta)=k\left(\theta, t_{\theta}\right)\left[g\left(\gamma\left(\theta, t_{\theta}\right)\right)-g\left(\theta^{0}\right)\right]$

and all the results released in the previous Section still hold, the linear integral transformation becoming a mixture of linear and nonlinear integral transformations:

$$
\begin{aligned}
& \eta(\mathbf{x}, \tau)=\int_{\Theta} h(\tau, \theta) \frac{t_{\theta}(p(\mathbf{x} ; \theta))}{p\left(\mathbf{x} ; \theta^{0}\right)} d \theta, \\
& \Gamma_{h}(\tau)=\int_{\Theta} h(\tau, \theta) k\left(\theta, t_{\theta}\right)\left[g\left(\gamma\left(\theta, t_{\theta}\right)\right)-g\left(\theta^{0}\right)\right] d \theta .
\end{aligned}
$$

At first sight, the proposed rationale does not seem appealing, since a non-linear transformation of type (9) is unlikely to exist whatever the form of the p.d.f., although the linear transformation of the LR (6) is always possible. Fortunately, it is applicable to a subset of $M$-dimensional complex circular Gaussian p.d.f.:

$$
p(\mathbf{x} ; \boldsymbol{\theta})=p(\mathbf{x} ; \mathbf{m}(\boldsymbol{\theta}), \mathbf{C}(\boldsymbol{\theta}))=\frac{e^{-(\mathbf{x}-\mathbf{m}(\boldsymbol{\theta}))^{H} \mathbf{C}(\boldsymbol{\theta})^{-1}(\mathbf{x}-\mathbf{m}(\boldsymbol{\theta}))}}{\pi^{M}|\mathbf{C}(\boldsymbol{\theta})|}
$$

Indeed, the transformation $t_{q}(y)=y^{q}$ can be applied to the observation model resulting from a mixture of deterministic and stochastic signals in presence of Gaussian interference [5]. In this case $\mathbf{m}(\boldsymbol{\theta})=\mathbf{m}(\varepsilon), \mathbf{C}(\boldsymbol{\theta})=\boldsymbol{\Psi}(\boldsymbol{\zeta}) \mathbf{C}_{\mathbf{s}} \boldsymbol{\Psi}(\boldsymbol{\zeta})^{H}+\mathbf{C}_{\mathbf{n}}$, $\boldsymbol{\theta}=\left[\varepsilon^{T}, \boldsymbol{\zeta}^{T}, \operatorname{vec}\left(\mathbf{C}_{\mathbf{s}}\right)^{T}, \operatorname{vec}\left(\mathbf{C}_{\mathbf{n}}\right)^{T}\right]^{T}$.

\section{CONJECTURAL LOWER BOUNDS}

Although initially introduced by resorting to the covariance inequality, the Bayesian bounds of the Weiss-Weinstein family have been lately revisited by authors in [6] who have shown that these bounds are also solutions of a norm minimization under linear constraints (see [6]§III.B) analogous to the one introduce in section 2. Therefore any deterministic lower bounds have a corresponding Bayesian bound: Cramér-Rao bound, Bhattacharyya bound, HammersleyChapman-Robbins bound, .... Our idea is to argue from analogy from the Bayesian bounds towards the deterministic bounds to explore new possible bounds. As an example, in the case of a single

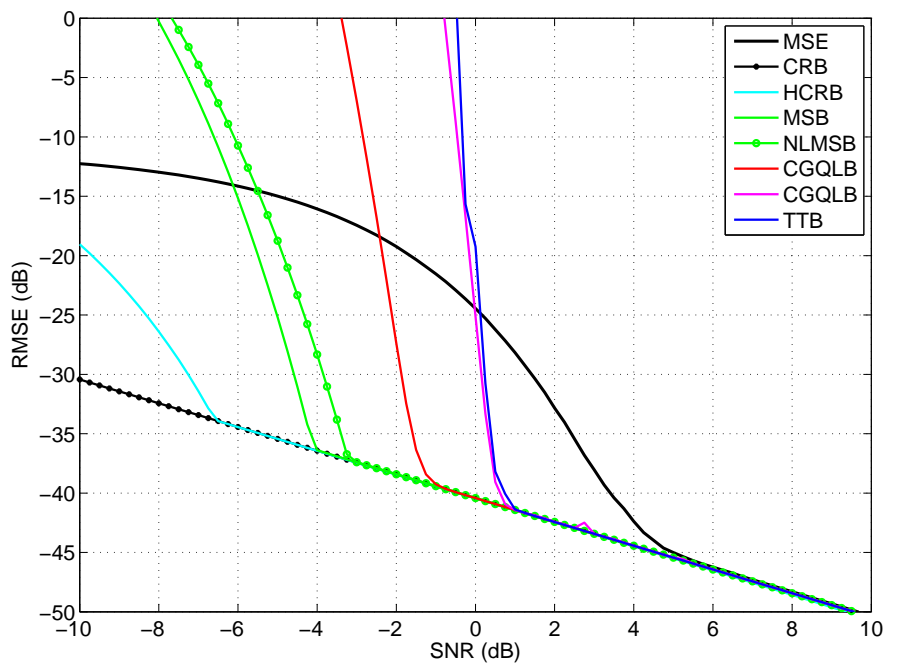

Fig. 1. Comparison of MSE lower bounds versus SNR $(M=8, \theta=0)$

unknown parameter $\theta$ - for sake of simplicity -, the Bayesian WeissWeinstein bound is associated with the linear constraint:

$$
\begin{array}{r}
\iint_{\Theta, \Omega}(\widehat{\theta}(\mathbf{x})-\theta)\left[\frac{p(\mathbf{x}, \theta+\delta)^{q}}{p(\mathbf{x}, \theta)^{q}}-\frac{p(\mathbf{x}, \theta-\delta)^{1-q}}{p(\mathbf{x}, \theta)^{1-q}}\right] p(\mathbf{x}, \theta) d \mathbf{x} d \theta \\
=-\delta \iint_{\Theta, \Omega} \frac{p(\mathbf{x}, \theta-\delta)^{1-q}}{p(\mathbf{x}, \theta)^{1-q}} p(\mathbf{x}, \theta) d \mathbf{x} d \theta
\end{array}
$$

where $p(\mathbf{x}, \theta)=p(\mathbf{x} \mid \theta) p(\theta)=p(\mathbf{x} ; \theta) p(\theta), q \in[0,1]$. The corresponding linear constraint for deterministic estimation is (drawn from examples in [6]§III.B):

$$
\begin{array}{r}
\int_{\Omega}\left(\widehat{\theta^{0}}(\mathbf{x})-\theta^{0}\right)\left[\frac{p\left(\mathbf{x} ; \theta^{0}+\delta\right)^{q}}{p\left(\mathbf{x} ; \theta^{0}\right)^{q}}-\frac{p\left(\mathbf{x} ; \theta^{0}-\delta\right)^{1-q}}{p\left(\mathbf{x} ; \theta^{0}\right)^{1-q}}\right] p\left(\mathbf{x} ; \theta^{0}\right) d \mathbf{x} \\
=-\delta \int_{\Omega} \frac{p\left(\mathbf{x} ; \theta^{0}-\delta\right)^{1-q}}{p\left(\mathbf{x} ; \theta^{0}\right)^{1-q}} p\left(\mathbf{x} ; \theta^{0}\right) d \mathbf{x}
\end{array}
$$

leading to the deterministic Weiss-Weinstein bound (WWB):

$$
M S E_{\theta^{0}}\left[\widehat{\theta^{0}}\right] \geq \sup _{q, \delta}\left\{\frac{\delta^{2} E_{\theta^{0}}\left[\frac{p\left(\mathbf{x} ; \theta^{0}-\delta\right)^{1-q}}{p\left(\mathbf{x} ; \theta^{0}\right)^{1-q}}\right]^{2}}{E_{\theta^{0}}\left[\left(\frac{p\left(\mathbf{x} ; \theta^{0}+\delta\right)^{q}}{p\left(\mathbf{x} ; \theta^{0}\right)^{q}}-\frac{p\left(\mathbf{x} ; \theta^{0}-\delta\right)^{1-q}}{p\left(\mathbf{x} ; \theta^{0}\right)^{1-q}}\right)^{2}\right]}\right\}
$$

The WWB (13) is a bound for estimators satisfying (12). The conjecture is that unbiased estimators satisfy (12) as well. It is true where $q=0$ or $q=1$ since then (12) amounts to the HammersleyChapman-Robbins constraint. Unfortunately so far, we have not been able to prove that (12) derives from the mixture of integral linear and non-linear transformations of the unbiasedness constraint. And yet, but nevertheless simulations performed for the single tone threshold analysis clearly shows that the WWB (13) is a very tight bound for unbiased estimators; at least in this application case. 


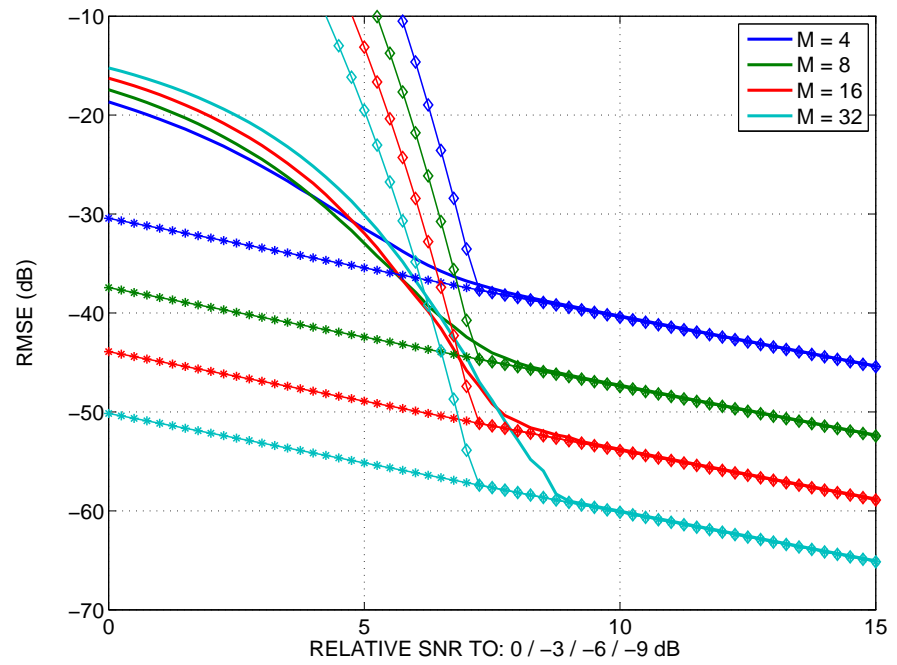

Fig. 2. MSE, CRB and WWB (Sup over $q \in[0,1])$ versus SNR

\section{CONCLUSION: SINGLE TONE THRESHOLD ANALYSIS}

A reference problem in threshold analysis is the estimation of a single tone $\theta \in]-0.5,0.5$ [ for a deterministic observation model:

$$
\begin{gathered}
\mathbf{x}=a \boldsymbol{\psi}\left(\theta^{0}\right)+\mathbf{n}, \quad \boldsymbol{\psi}(\theta)=\left[1, \ldots, e^{j(M-1) 2 \pi \theta}\right]^{T} \\
p(\mathbf{x} ; \theta)=\frac{e^{-\|\mathbf{x}-a \psi(\theta)\|^{2}}}{\pi^{M}}
\end{gathered}
$$

where $a^{2}$ is the known SNR $(a>0)$ and $\mathbf{n}$ is a complex circular Gaussian noise, with zero mean and a known covariance matrix $\mathbf{C}_{\mathbf{n}}=\mathbf{I}$. In the simulations:

- $\delta \in] 0,0.5\left[, \widehat{\theta}_{M L}=\max _{\theta}\left\{\operatorname{Re}\left[\boldsymbol{\psi}(\theta)^{H} \mathbf{x}\right]\right\}\right.$.

- the HCRB [2] is the simplest approximation of the BB (5) based on 2 test-points $\boldsymbol{\theta}^{2}=\left(\theta^{0}, \theta^{0}+\delta\right)^{T}+$ supremum on $\delta$,

- the MSB [2] is the simplest approximation of the BB based on 3 test-points $\boldsymbol{\theta}^{3}=\left(\theta^{0}, \theta^{0}+\delta, \theta^{0}-\delta\right)^{T}+$ supremum on $\delta$,

- the NLMSB [5] is the nonlinear generalisation (10) of the MSB based on 3 test-points + supremum on $\delta$ and $q \in] 0.5,2[$,

- the CGQLB [2] is the generalization of the CRB based on 3 testpoints $\boldsymbol{\theta}^{3}=\left(\theta^{0}, \theta^{0}+\delta, \theta^{0}-\delta\right)^{T}+$ supremum on $\delta$,

- the TTB [3] is the combination of $\operatorname{CRB}\left(\theta^{0}\right)$ and of (5) where $N=1024, K=32$ and $\mathbf{H}_{K}$ is an ad hoc submatrix of FFT matrix of dimension $N$.

All these lower bounds are displayed on figure (1) and compared with the MSE of the MLE estimator $\left(5 \times 10^{5}\right.$ trials) for $M=8$ and $\theta^{0}=0$. The first occurrence of the CGQLB is obtained for $\delta$ lying on a discretization of $]-0.5,0.5$ [ with a step of $1 / 1024$. The second one is obtained for a step of $1 /(1024 * 128)$. The purpose of the 2 cases is to show that it is generally difficult to compare tightness of bounds which are based on subsets of constraints that are not included one in each other. For each bound, tightness may depend on specific optimization parameters.

Additionally, the tightness of CGQLB and TTB (or any existing bound) could be improved by updating their associated linear constraints with the non-linear transformation (10) as we did for the MSB, which is a topic for future work.

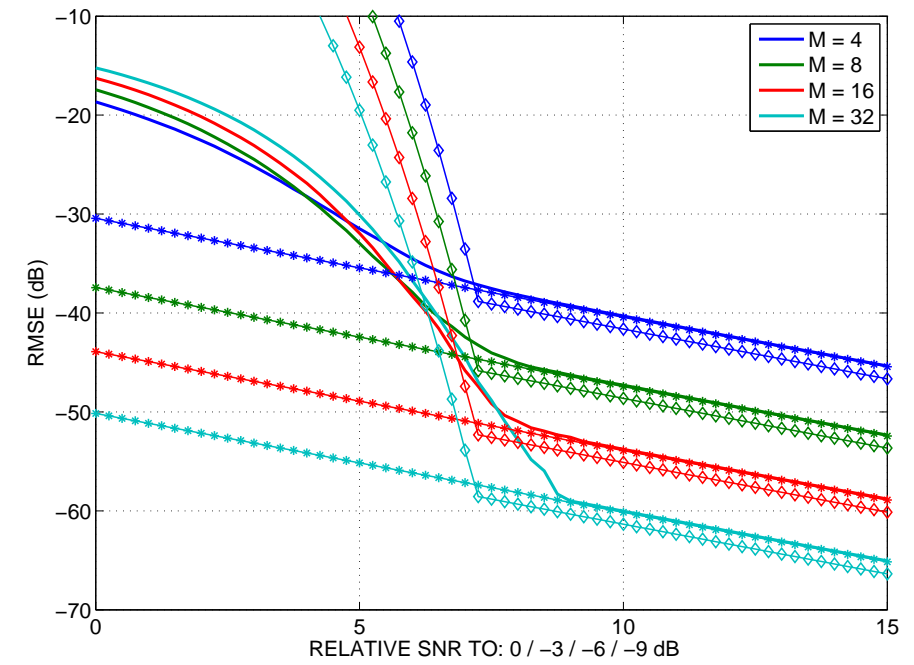

Fig. 3. MSE, CRB and WWB $\left(q=\frac{1}{2}\right)$ versus SNR

Finally, a much more impartial criterion could be the computation time (and possibly the memory load).

Nevertheless, all these bounds seem to provide a still too coarse prediction of the SNR threshold value (underestimated by at least $5 \mathrm{~dB})$, an imperfection mostly compensated by the WWB (13) as shown on figure (2). This figure clearly shows that the WWB is not only a lower bound for unbiased estimators whatever the value of $M$ (also checked for $M=2,64,128$ ), but it is an extremely tight lower bounds, far tighter that all the existing ones.

Moreover, the deterministic WWB seems to share the same property as its Bayesian analogue one, i.e. to be nearly the tightest for $q=0.5$ as shown on figure (3). Under that form, the WWB is as simple to implement as the HCRB.

Such a simple and tight bound really deserves to be derived!

\section{REFERENCES}

[1] E.W. Barankin, "Locally best unbiased estimates", Ann. Math. Stat., vol. 20, no. 4, pp. 477-501, 1949.

[2] E. Chaumette, J. Galy, A. Quinlan, P. Larzabal, "A New Barankin Bound Approximation for the Prediction of the Threshold Region Performance of Maximum-Likelihood Estimators", IEEE Trans. on SP, vol. 56, no. 11, pp. 5319-5333, Nov. 2008

[3] K. Todros and J. Tabrikian, "A new lower bound on the meansquare error of unbiased estimators", in Proc. IEEE Int. Conf. Acoust., Speech, Signal Process., pp. 3913-3916, 2008

[4] H.L. Van Trees, Detection, Estimation and Modulation Theory, Part 1. New York: Wiley, 1968.

[5] E. Chaumette, A. Renaux, P. Larzabal, "Lower bounds on the mean square error derived from mixture of linear and non-linear transformations of the unbiasness definition", in Proc. IEEE Int. Conf. Acoust., Speech, Signal Process., pp. 3045-3048, 2009

[6] A. Renaux, P. Forster, P. Larzabal, C.D. Richmond, A. Nehorai, "A Fresh Look at the Bayesian Bounds of the Weiss-Weinstein Family", IEEE Trans. on SP, vol. 56, no. 11, pp. 5334-5352, Nov. 2008 